\title{
Chemistry aided nuclear physics studies
}

\author{
Julia Even ${ }^{\mathrm{a}}$ \\ KVI - CART, University of Groningen, 9747 AA Groningen, The Netherlands
}

\begin{abstract}
Studies of the superheavy elements bring several challenges through low production yields, short half-lives, and high background rates. This paper describes the possibilities of chemical separations as techniques to overcome the background problematic and to investigate the nuclear properties of the heaviest nuclides.
\end{abstract}

\section{Introduction}

The transuranium elements up to nobelium (No, $Z=102$ ) have been discovered applying radiochemical separation methods (see, for example, reference [1]). The discoveries of the heaviest actinoide element lawrencium ( $\mathrm{Lr}, Z=103)$ and the first transactinoide rutherfordium (Rf, $Z=104$ ) have been achieved through the application of nuclear physics techniques and followed shortly after by radiochemical separations. With increasing proton number, the production cross sections and the half-lives decrease. Thus, from the discovery of dubnium ( $\mathrm{Db}, Z=105$ ) on, physics techniques were the driving forces for the discovery of new elements. In the early eighties, the area of superheavy elements at electromagnetic separators started with the discovery of bohrium (Bh, $Z=107)$, hassium (Hs, $Z=108)$, and meitnerium (Mt, $Z=109$ ) at SHIP at GSI, Darmstadt (see contribution of S. Hofmann in this proceedings, and reference [2]). Although physics techniques dominate the research on heavy elements in our days, chemists are still actively involved in this field. Besides studies of the chemical properties, chemical separations play a role in nuclear physics studies. In this paper, examples will be given how the combination of chemistry and physics can overcome recent challenges.

\section{Identification of the proton number}

The elements flerovium ( $\mathrm{Fl}, Z=114$ ) up to element $Z=118$ have been so far only synthesized in ${ }^{48} \mathrm{Ca}$-induced fusion reactions with actinoide targets [3]. However, an unambiguous identification of the proton number of these elements could still not be achieved. All these elements decay into previously unknown spontaneous fission isotopes. Thus, there is no direct link to the well-known part of the nuclear chart. In case of ${ }^{288} 115$, the $\alpha$-decay chains end in a long-lived nuclide which decays through spontaneous fission. This long-lived fission nucleus was assigned to ${ }^{268} \mathrm{Db}$ or after decay through electron capture to ${ }^{268} \mathrm{Rf}$. One option to identify the proton number of ${ }^{288} 115$ is the chemical isolation and identification of the daughter nuclide ${ }^{268} \mathrm{Db}$ or ${ }^{268} \mathrm{Rf}[4,5]$. For this approach, ${ }^{288} 115$ was produced in the reaction ${ }^{243} \mathrm{Am}\left({ }^{48} \mathrm{Ca}, 3 n\right)$ and implanted in a copper block. After irradiation, the upper copper layer was dissolved, elements of group four and group five were

\footnotetext{
a e-mail: j.even@rug.nl
}

(C) The Authors, published by EDP Sciences. This is an Open Access article distributed under the terms of the Creative Commons Attribution License 4.0 (http://creativecommons.org/licenses/by/4.0/). 
separated through ion-exchange chromatography. Samples of the chromatography fractions were prepared for spontaneous fission and $\alpha$ spectroscopy. Each sample was measured for 960 hours. In the group four and group five fraction, several fission events were observed, which were assigned to the decay of long-lived $\mathrm{Rf}$ or $\mathrm{Db}$ isotopes $[4,5]$. These results were yet not included in the assignment of element 115 by the joint working party of IUPAP and IUPAC [6]. Their report notes that a new chemical separation scheme for $\mathrm{Db}$ and $\mathrm{Rf}$ was applied. In the past, Db and Rf chemistry had already been studied with the isotopes ${ }^{262,263} \mathrm{Db}$ and ${ }^{257,261} \mathrm{Rf}$, which were directly produced in fusion-evaporation reactions [7, 8]. Relativistic effects influence the chemical behaviour of $\mathrm{Db}$ and Rf. In the future, a chemical system which has been already used to study transactinoides directly produced in fusion evaporation reaction, should be used for the identification of the proton number. Thus, miss-interpretation of data due to unknown or unexpected chemical properties of these elements can be excluded.

\section{In-situ gas-phase chemistry}

Besides ion-exchange chromatography also gas-phase chemical techniques are playing an important role in superheavy element research. Especially, studies of isotopes in the vicinity of the neutron sub-shell closure around $N=162$ and the proton sub-shell closure $Z=108$ can benefit from gas-phase chemical techniques. These nuclides are primarily accessible in reactions with relatively light projectiles (such as ${ }^{26} \mathrm{Mg}$ or ${ }^{22} \mathrm{Ne}$ ) and actinoide targets. Studies of reaction products of these reactions are challenging for an electromagnetic separator as the target transfer products have almost the same magnetic rigidity as the fusion-evaporation products of interest. This leads to a high background rate in the focal plane. Thus, alternative or additional separation methods need to be found. One possibility is selective in-situ gasphase chemistry.

\subsection{Volatile group 8 tetroxides}

In case of hassium (Hs, $Z=108$ ), the separation, and transport as volatile hassium tetroxide $\left(\mathrm{HsO}_{4}\right)$ has been established. Hassium tetroxide was first synthesized and investigated in an experiment focusing on its chemical properties [9]. ${ }^{269} \mathrm{Hs}$ was produced in the reaction ${ }^{248} \mathrm{Cm}\left({ }^{26} \mathrm{Mg}, 3 n\right)$, and thermalized directly behind the target in a recoil chamber which contained a helium-oxygen mixture. Hassium reacts with the oxygen and forms like its lighter homologue element osmium a volatile tetroxide. Within a few seconds, $\mathrm{HsO}_{4}$ can be transported in a gas stream out of the recoil chamber through capillaries to detector devices. $\mathrm{HsO}_{4}$ can be deposited at low temperatures on the surface of an $\alpha$-detector.

In contrast to Hs the nuclear by-products do not form volatile oxides and are thus not transported. Only noble gases and volatile elements reach the detector through the gas stream. Chemistry is thus more efficient than any electromagnetic separator. However, the technique is limited by the half-life of the Hs isotopes. Only isotopes which live a few seconds can be investigated.

$\mathrm{HsO}_{4}$ has successfully applied to separate $\mathrm{Hs}$ isotopes for studies near the neutron sub-shell closure at $N=162$. To optimize spectroscopy of Hs, the thermochromatography detector COMPACT was build [10,11]. The detector consists of two arrays of 32 PIN diodes each. These two arrays form a 0.6-mm wide channel with a negative temperature gradient. The transport gas enters this channel at the warm side and leaves the detector through the cold end. $\mathrm{HsO}_{4}$ is deposited in the middle of this channel at around $-45^{\circ} \mathrm{C}$. The detection efficiency for $\alpha$ particles is about $80 \%$ [10]. $\mathrm{HsO}_{4}$ was used to study the excitation function of the reaction ${ }^{248} \mathrm{Cm}\left({ }^{26} \mathrm{Mg}, 3-5 n\right)$ [10-12]. With this approach, the isotopes ${ }^{270} \mathrm{Hs}$ and ${ }^{271} \mathrm{Hs}$ were discovered [10, 11]. Also the reaction ${ }^{238} \mathrm{U}\left({ }^{36} \mathrm{~S}, 4 n\right)$ has been investigated [13]. 


\subsection{Metal carbonyl complexes}

To study other nuclei with the COMPACT detector, new suitable chemical systems have to be investigated. Recently, the in-situ chemistry of metal carbonyl complexes has been explored [14-18] and it is still under investigation (see contribution R. Eichler et al. in this proceedings). Group 6 and group 8 elements were known to form volatile complexes with carbon monoxide. However, these complexes are not stable at temperatures of a few hundred degrees Celsius [18]. Direct synthesis in a recoil chamber behind the target is not possible due to the presence of a plasma induced by the primary beam. The fusion products need to be pre-separated from the primary beam to allow the formation of carbonyl complexes. In a first experiment at the gas-filled separator TASCA, volatile complexes of short-lived isotopes of tungsten, rhenium, osmium, and iridium were synthesized and transported in the gas stream to the chromatography detector COMPACT $[14,16]$. These studies demonstrated the potential that carbonyl chemistry bears for superheavy element research. In a follow-up experiment at the GARIS separator at RIKEN, the first carbonyl complex of a superheavy element was synthesized - seaborgium hexacarbonyl [15]. Seaborgium could be isolated from the huge amount of target-like transfer products which also reached the focal plane of GARIS. It was transported in the gas stream to the chromatography detector. Figure 1 shows a sum spectrum of all $64 \alpha$ detectors in the COMPACT array [17]. As it can be clearly seen, the interesting energy region above $8 \mathrm{MeV}$ is background free. The $\alpha$ event at $8.56 \mathrm{MeV}$ was followed $214 \mathrm{~s}$ later by the coincident detection of two fission fragments at $11 \mathrm{MeV}$ and $74 \mathrm{MeV}$, respectively, in the same detector pair. This experiment was the first experiment which was sensitive for such a long correlation time. The observed decay chain of ${ }^{265} \mathrm{Sg}$ which is shown in Fig. 1 gives a hint for new decay properties of ${ }^{261} \mathrm{Rf}$ [17]. Two isomeric states of ${ }^{261} \mathrm{Rf}$ have been reported in literature [19, 20]. ${ }^{261 a} \mathrm{Rf}$ decays through $\alpha$ emission with a half-life of $T_{1 / 2}=70 \mathrm{~s}$. ${ }^{261 b} \mathrm{Rf}$ is comparably short lived with $T_{1 / 2}=$ $1.9 \mathrm{~s}$ and decays through $\alpha$ emission or spontaneous fission. Observations in the carbonyl chemistry experiment led to the speculation that ${ }^{261 a} \mathrm{Rf}$ might have an unknown spontaneous fission branch, or that there might be a ${ }^{261} \mathrm{Rf}$ isomer. This isomer may decay through electron capture into ${ }^{261} \mathrm{Lr}$ which has a half-life of $T_{1 / 2}=39 \mathrm{~min}$. Further investigations are required.

The studies of carbonyl complexes demonstrated their potential for low-background spectroscopy of seaborgium. Furthermore, the studies of the lighter homologue elements of group 7, 8, and 9 showed that bohrium, hassium, and meitnerium are accessible using carbonyl chemistry.

\section{Chemical reactions in gas catchers}

Gas-catchers are commonly used in nuclear physics experiments to thermalize recoiling ions in an inert gas atmosphere and extract these through electric fields to prepare samples for precision studies. Generally, the gas in a gas catcher is kept as clean as possible. Gas catchers have been already implemented at the heavy element separators SHIP, BGS, and GARIS to couple these to mass spectrometers or mass analyzers [21-23]. So far chemical reactions in a gas catcher have not been established at these facilities.

Nevertheless, ion chemistry can be used as a powerful tool to prepare samples for nuclear physics studies. At the Low Energy Beam and Ion Trap (LEBIT) facility at the National Superconducting Cyclotron Laboratory (NSCL), chemistry has already applied to separate nuclear isobars. LEBIT is a Penning-trap facility built for high precision mass measurements of rare isotopes produced by fast-beam fragmentation [24]. LEBIT consists of a gas catcher in which the ions delivered from the fragment separator are thermalized. Subsequently, a set 


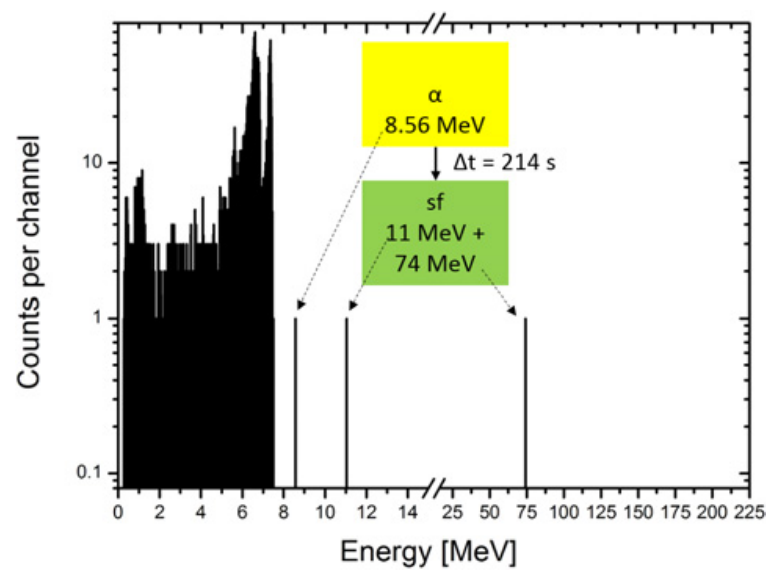

Figure 1. Sum spectrum of all $64 \alpha$ detectors of the COMPACT detector array. The spectrum was collected while $4.35 \cdot 10^{17}{ }^{22} \mathrm{Ne}$ particles hit the target. The spectrum is free from background activity in the interesting energy range above $7.5 \mathrm{MeV}$. The $\alpha$ peaks at energies below $7.5 \mathrm{MeV}$ originate from the decay of ${ }^{219} \mathrm{Rn}$ and its daughter isotopes ${ }^{215} \mathrm{Po}$ and ${ }^{211} \mathrm{Bi}$. A ${ }^{227} \mathrm{Ac}$-emanation source was implemented into the gas loop for continous calibration of the COMPACT detectors. Graphic according to reference [17].

of radio-frequency quadrupole (RFQ) structures guides the ions through different pressure regimes and the species of interest is selected in an RFQ section which operates as a mass filter. Then the ions pass a beam cooler and buncher before they are injected into the Penning trap for mass measurements.

For mass measurements of ${ }^{32} \mathrm{Si}$ at LEBIT, chemical reactions in a gas catcher were applied to prepare clean samples [25]. ${ }^{32} \mathrm{Si}$ was produced in in-flight fragmentation of ${ }^{40} \mathrm{Ar}$ and extracted along with ${ }^{32} \mathrm{~S}$ and ${ }^{16} \mathrm{O}_{2}$ from the gas catcher. The isobaric contamination was several orders of magnitude higher. Thus, element-selective separation was required. Therefore, water vapor was added to the gas in the stopping cell. Si formed ${ }^{32} \mathrm{SiOH}\left(\mathrm{H}_{2} \mathrm{O}\right)^{+}$ with a molecular mass of $67 \mathrm{u}$ while $\mathrm{S}$ formed ${ }^{32} \mathrm{SO}_{2} \mathrm{H}^{+}$with a molecular mass of $65 \mathrm{u}$, and $\mathrm{O}_{2}$ did not react. Thus, these species could be separated in a mass analyzer behind the gas cell. The molecular bonds were subsequently broken in the beam cooler and buncher through collisions with the gas before injection of the ions into the Penning trap [25].

For mass measurements of the heaviest elements, so far an extra separation step was not required. However, this method will become interesting for superheavy chemistry research. Here physics techniques can aid chemistry studies. Until now, the interpretation of the chemistry studies strongly relies on the support from theoretical predictions and studies of the lighter homologue elements. The structure of the chemical compounds cannot unambiguously be determined. Thus, the combination of gas-chemistry with more sophisticated methods such as mass spectrometry would be beneficial. The approach of chemical reactions in a gas catcher combined with mass measurements appears feasible. A good system to start with are the transition metal carbonyl complexes. In mass measurements of Fe and Co at LEBIT, it was observed that Fe and Co formed mono-, bi-, and even tri-carbonyl complexes with small impurities of carbon monoxide in the buffer gas [24]. Controlling the amount of carbon monoxide in the buffer gas will open the door for kinetic studies of the carbonyl complex formation of even the transition metals of the $7^{\text {th }}$ period of the periodic table. 


\section{Conclusion and outlook}

Although the period of chemically driven element discovery passed, nuclear physics experiments can still benefit from chemical techniques. Chemical studies can be used to identify the proton number of an isotope. Furthermore, fast gas-phase transport of volatile species can be applied for background free decay spectroscopy. Group 8 tetroxide and the volatile transition metal carbonyl complexes are powerful chemical separators. Currently, new detectors are under development to improve the detection efficiency and allow $\alpha, \beta$, and $\gamma$ coincidence measurements aided by gas-phase chemistry [26]. This new detector type will have a sandwich structure. The inner chromatography channel consists of an $\alpha$-detector array followed by layers of detectors sensitive for electrons and photons.

However, also superheavy element chemistry can benefit from developments in experimental nuclear physics. Ion-chemistry combined with single molecule mass spectrometry will open the door to unambiguously identify the structure of chemical compounds of the heaviest elements. It will open the door for studies of chemical reaction kinetics. A wise choice of combination of the different experimental methods will lead to many interesting discoveries in the future.

The author acknowledges support through the Rosalind Franklin fellowship program of the University Groningen co-funded by the EU.

\section{References}

[1] Y. Nagame and M. Hirata, Radiochim. Acta 99, 377 (2011).

[2] G. Münzenberg and K. Morita, Nucl. Phys. A944, 3 (2015).

[3] Y.T. Oganessian and V.K. Utyonkov, Nucl. Phys. A944, 62 (2015).

[4] D. Schumann et al., Radiochim. Acta 93, 727 (2005).

[5] N.J. Stoyer et al., Nucl. Phys. A787, 388 (2007).

[6] P.J. Karol et al., Pure and Appl. Chem. 88, 139 (2016).

[7] J.V. Kratz, Radiochim. Acta 99, 477 (2011).

[8] M. Schädel, Phil. Trans. R. Soc. A 373, 20140191 (2015).

[9] C.E. Düllmann et al., Nature 418, 859 (2002).

[10] J. Dvorak et al., Phys. Rev. Lett. 97, 242501 (2006).

[11] J. Dvorak et al., Phys. Rev. Lett. 100, 132503 (2008).

[12] J. Dvorak et al., Phys. Rev. C 79, 037602 (2009).

[13] R. Graeger et al., Phys. Rev. C 81, 061601 (2010).

[14] J. Even et al., Inorg. Chem. 51, 6431 (2012).

[15] J. Even et al., Science 345, 1491 (2014).

[16] J. Even et al., Radiochim. Acta 102, 1093 (2014).

[17] J. Even et al., J. Radioanal. Nucl. Chem. 303, 2457 (2015).

[18] I. Usoltsev et al., Radiochim. Acta 104, 141 (2016).

[19] H. Haba et al., Phys. Rev. C 83, 034602 (2011).

[20] H. Haba et al., Phys. Rev. C 85, 0246411 (2012).

[21] M. Block et al., Eur. Phys. J. A 50, 49 (2005).

[22] P. Schury et al., Nucl. Instr. Meth. B317, 537 (2013).

[23] J.M. Gates, these proceedings.

[24] R. Ferrer et al., Phys. Rev. C 81, 044318 (2010).

[25] A.A. Kwiatkowski et al., Phys. Rev. C 80, 051302 (2009).

[26] A. Di Nitto et al., in GSI Scientific Report 2014, GSI Report 2015-1, p. 184. 